\title{
Exploring IoT protocols through the Information-Centric Networking's lens
}

\author{
Salvatore Signorello ${ }^{1}$, Radu State ${ }^{1}$, and Olivier Festor ${ }^{2}$ \\ 1 SnT, University of Luxembourg, \\ \{salvatore.signorello, radu.state\}@uni.lu \\ 2 INRIA Nancy Grand Est \\ \{olivier.festor\}@inria.fr
}

\begin{abstract}
Information-Centric Networking (ICN) advocates a cleanslate redesign of the Internet network layer through a major shift from a host-to-host communication model to a content-based one. Hence ideally, current ICN propositions and implementations, for instance NDN (Named Data Networking), should replace the IP layer. Indeed none of these already provide a full-fledged solution, due to the big challenges that their deployment at a global scale implies. Nevertheless ICN's ambitious goal could be a plausible short-term step for networks at the Internet's borders, where several layer-2 technologies populate the Internet of Things ecosystem. Those domains still need an in-depth analysis of the potential of the ICN solutions as well as of the concrete mechanisms to deploy them. Thus in the following, we've sketched out our research path to find out common layer- 2 requirements for the future Internet's network-layer.
\end{abstract}

Keywords: ICN, network-layer, NDN, IoT

\section{Introduction}

The Internet's architecture has notably served our needs so far, even if, since its original design, the requirements for a global communication infrastructure have radically changed [1]. Nowadays many researchers finally have agreed on the concrete need for a redesign of the network architecture to improve its efficiency and to assure it a longer life. A novel networking approach, called InformationCentric Networking [2], suggests to shift the model of communication used by the Internet from a host-to-host dialogue to a network-driven search for sources of information.

Since the coming out of PARC with Van Jacobson's google-talk [15] and the subsequent publication of the seminal paper on Content-Centric Networking [4] have spread the idea of Information Centric Networking, several advances have been done in the field. As counterpart, there are still several critical research challenges [16] that need to be addressed by ICN and those unanswered questions slow down its adoption.

Today the ICN community eagerly seeks use-cases to showcase the advantages 
this novel network paradigm might bring in real-life scenarios [18]. To this regard, the ICN data-oriented approach appeals to the Internet of Things (IoT) domain as the perfect glue for internetworking the IoT islands. Nevertheless previous research works have just showcased the feasibility of adopting ICN for some specific IoT scenarios, e.g., BASs [5,6], MANETs [7, 8], vehicular networks $[9$, 10] and sink-centric WSNs [11-13]. Each of those attempts relies on ad-hoc assumptions and none of them identify a common set of services that an ICN layer should expose to the IoT's layer-2 technologies. This lack of consensus on few core design principles implies that current ICN solutions often have to be heavily tailored for the deployment in IoT scenarios.

\section{Background on Named Data Networking}

The NDN architecture [3] is the ICN instantiation targeted for our study. The NDN communication model is receiver-driven and it is governed by two different named network packets, the Interest and the Data, and by three data structures, the Forwarding Information Base (FIB), the Pending Interest Table (PIT) and the Content Store (CS). The communication is started by information consumers that issue Interests to request contents. Then the network routes by name the Interests (matching FIB entries held by the network devices) towards one or more content providers (either the original source or devices that have cached a copy in their CS). Finally the Data is routed back to the requester, following the trail left (the PIT entries) by the corresponding Interest into the traversed network devices.

NDN features several design choices that appeal to the IoT. It provides innetwork caching and content-based security (every packet carries a digital signature) that might reduce the duty cycle of constrained-resources devices, as long as the information they've disseminated will be valid. It provides flow balance mechanisms, matching an Interest to a single Data packet, that might reduce the overall traffic and so the demand for computation into the network devices. It allows to define human-readable naming schemes to address the network's contents, and this might ease the services and objects discovery which is crucial in IoT dense environments. Moreover NDN performs multi-path namebased forwarding of network packets, that is, a network device can forward a request to multiple next hops based both on some information it holds and on the request's name. The forwarding logic can be further specialized through the plug-in of user-defined software modules, called strategies, to perform custom forwarding decisions.

\section{$3 \quad$ Research statement}

The Internet was originally devised to deliver packets to fixed destination addresses and now it is expected to serve billions of mobile devices jumping between different networks. Any clean-slate attempt to evolve the Internet's architecture 
has to accommodate both emerging and consolidated technologies in a fresh design. So any conscious advocate of ICN should ask himself: "Is ICN ready to welcome the Internet-of-Things?"

The success of the Internet Protocol stems from the neat layered design of the whole network architecture. At the Internet's rise the main requirements for a global network were translated into IP's packet format fields. For instance, the need of internetworking was barely encoded into the IP datagram format as fragmentation fields and, not surprisingly, it is still of immense relevance. The history of computer networks has taught us that there will always be layer- 2 technologies with different requirements that need to inter-operate and this aspect has become more and more evident with the proliferation of network technologies throughout the last decade: WiFi, WiMAX, Bluetooth, LoWPANs standards, $4 \mathrm{G}$ and sooner its descendant $5 \mathrm{G}$.

ICN has potential features to welcome the heterogeneity of technologies that characterizes the IoT, but it has evidently not been designed to fulfill this objective. Therefore, we strive to enhance the design of current ICN solutions through the analysis of their deployment in scenarios where end-users join several networks at the same time. In figure 1 we illustrate one of the scenarios on target. We suppose to have an user with a laptop equipped with three different network interfaces. The user's laptop has an Ethernet interface plugged to a private company LAN. At the same time the user has joined a different ad-hoc WiFi network with some colleagues to do collaborative tasks. Finally, the user also has broadband Internet access, e.g., through either an usb-modem for cellular connection or PC-card for WiMAX access.

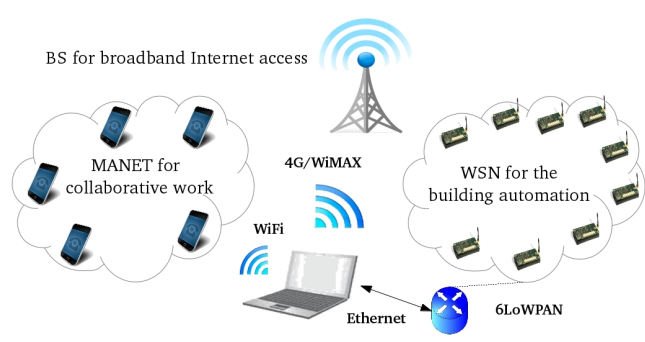

Fig. 1. Targeted use case scenario

One trivial use-case for the scenario depicted above could be the following: the user wants to fetch some content from the web and some of his colleagues might have already fetched the same; so if some local copies exist, the user would prefer to fetch them for many possible reasons, e.g., to save traffic, to have faster download times, to not worry about cellular connection instability. Each time the user's application make a call to a network primitive, the network level should try to fit out the best from the local resources and from the neighborhood. So the question is: "Are current ICN solutions really ready to serve to this need?" 


\section{Methodology and preliminary results}

Our research roadmap mainly targets the testing and the extension of the NDN's platform codebase [19]. However we use in parallel the network simulator ndnSim [14] based on the ns-3, a network simulator that offers modules modeling the most common layer-2 technologies.

We've tested how the plain NDN performs in simple scenarios wherein some nodes exhibit both WiFi and Ethernet connectivity. We've run some rounds of simulation over a three-level tree network topology, where all the leaf nodes fetch content from a single producer at the root of the tree, see figure 2. We've run the experiments for three distinct scenarios, namely labeled as Caching, WiFi, WiFi \& Caching. In the Caching scenario, all the tree nodes cache content, but none of them have WiFi connectivity. In the WiFi scenario, leaf nodes have $\mathrm{WiFi}$ and they're the only tree nodes that can cache contents. In the WiFi \& Caching scenario, leaf nodes have WiFi and all the tree nodes can cache content. In fig. 3 we've reported the average download time measured using different built-in ndnSim's forwarding strategies [17]. Surprisingly the average download time never decreases in presence of cached contents by the consumer's WiFineighbors, on the contrary it worsens. The latter condition happens because of the high collision rate produced by the intense broadcast WiFi traffic [20].

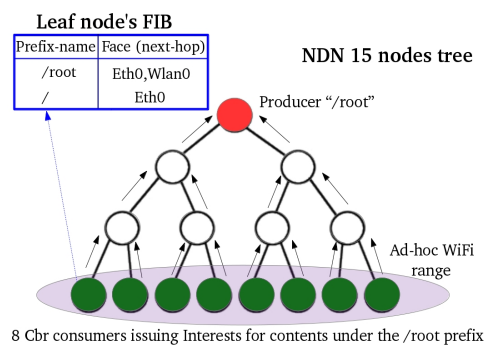

Fig. 2. Topology for preliminary tests

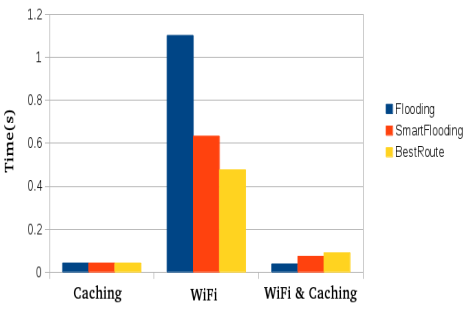

Fig. 3. Average download time

\section{Conclusion}

As expected, this first hands-on experience outlines that the NDN protocol, as it is, cannot run efficiently over different layer-2 without adaptation and thus it calls for a deeper investigation. As next step, we've started implementing a custom forwarding strategy into ndnSim, which prioritizes output interfaces according to their data-link layer. 


\section{References}

1. R. Braden, D. Clark, S. Shenker, J. Wroclawski: Developing a Next-Generation Internet Architecture. White paper, DARPA, July 2000.

2. B. Ahlgren, C. Dannewitz, C. Imbrenda, D. Kutscher, and B. Ohlman: A survey of Information-Centric Networking. IEEE Communications, vol. 50, no. 7, pp. 2636 , July 2012.

3. L. Zhang, A. Afanasyev, J. Burke, V. Jacobson, K. Claffy, P. Crowley, C. Papadopoulos, L. Wang, and B. Zhang: Named Data Networking. ACM SIGCOMM Computer Communication Review (CCR), July 2014.

4. V. Jacobson, D. K. Smetters, J. D. Thornton, M. F. Plass, N. H. Briggs, and R. L. Braynard: Networking named content. In International conference on Emerging networking experiments and technologies CoNEXT. ACM, 2009.

5. J. Burke, P. Gasti, N. Nathan, G. Tsudik: Securing Instrumented Environments over Content-Centric Networking: the Case of Lighting Control and NDN. In IEEE INFOCOM NOMEN Workshop, 2013.

6. J. Zhang, Q. Li, E. M. Schooler: iHEMS an Information-Centric approach to secure home energy management. In IEEE SmartGridComm, 2012.

7. Y. Yu et al.: Interest propagation in named data manets. In Proc. of IEEE ICNC, 2013, pp. 11181122.

8. M. Meisel, V. Pappas, L. Zhang: Ad Hoc Networking via Named Data. ACM MobiArch 2010.

9. L. Wang, A. Afanasyev, R. Kuntz, R. Vuyyuru, R. Wakikawa, L. Zhang: Rapid traffic information dissemination using named data. In Proc. of the 1st ACM workshop on Emerging Name-Oriented Mobile Networking Design-Architecture, Algorithms, and Applications, pages 712. ACM, 2012.

10. G. Grassi, D. Pasavento, G. Pau, R. Vuyyuru, R. Wakikawa, L. Zhang: VANET via Named Data Networking. In IEEE INFOCOM Workshop on Name Oriented Mobility (NOM), Toronto, Canada, April-May 2014.

11. M. Amadeo et al.: Named data networking: A natural design for data collection in wireless sensor networks. In Proc. of IEEE/IFIP Wireless Days, 2013, pp. 16.

12. N.-T. Dinh, Y. Kim: Potential of Information-Centric Wireless Sensor and Actor Networking. In IEEE International Conference on Computing, Management and Telecommunications (ComManTel), 2013.

13. J. Francois, T. Cholez, and T. Engel: CCN Traffic Optimization for IoT. In The 4th International Conf. on Network of the Future (NoF), 2013.

14. A. Afanasyev, I. Moiseenko, L. Zhang: ndnSIM: NDN simulator for NS-3. NDN Technical Report NDN-0005, Revision 2, October 2012

15. V. Jacobson: A new way to look at networking. Google Tech Talk, https://www . youtube. com/watch?v=oCZMoY3q2uM

16. ICN Research Challenges draft-kutscher-icnrg-challenges-02, http: //datatracker.ietf.org/doc/draft-kutscher-icnrg-challenges/

17. ndnSim's Forwarding Strategies, http://ndnsim.net/1.0/fw.html

18. Information-Centric Networking: Baseline Scenarios draft-irtf-icnrg-scenarios-03, https://datatracker.ietf .org/doc/draft-irtf-icnrg-scenarios/

19. The named-data networking project's website, http://named-data.net/

20. C. Campolo, A. Molinaro, C. Casetti, and C.-F. Chiasserini: An 802.11-based MAC Protocol for Reliable Multicast in Multihop Networks. In Proc. of Vehicular Technology Conference, april 2009, pp. 15. 